\title{
Bottom-Up Activation of the Vocal Motor Forebrain by the Respiratory Brainstem
}

\author{
Robin C. Ashmore, ${ }^{1,2}$ Jessica A. Renk, ${ }^{2}$ and Marc F. Schmidt ${ }^{1,2}$ \\ ${ }^{1}$ Department of Biology, and ${ }^{2}$ Neuroscience Graduate Group, University of Pennsylvania, Philadelphia, Pennsylvania 19104
}

\begin{abstract}
Brainstem motor structures send output commands to the periphery and are dynamically modulated by telencephalic inputs. Little is known, however, about ascending brainstem control of forebrain motor structures. Here, we provide the first evidence for bottom-up activation of forebrain motor centers by the respiratory brainstem. We show that, in the avian vocal control system, activation of the brainstem inspiratory nucleus paraambigualus (PAm), a likely homolog of the mammalian rostral ventral respiratory group, can drive neural activity bilaterally in the forebrain vocal control nuclei HVC (used as a proper name) and the robust nucleus of the arcopallium (RA). Furthermore, this activation is abolished by lesions of nucleus uvaeformis (Uva), a thalamic nucleus necessary for song production. We identify a type of bursting neuron within PAm whose activity is correlated, in an Uva dependent manner, to bursting activity in RA, rather than to the respiratory rhythm, and is robustly active during the production of stimulus evoked vocalizations. Because this ascending input results in cross-hemisphere activation, our results suggest a crucial role for the respiratory brainstem in coordinating forebrain motor centers during vocal production.
\end{abstract}

Key words: respiration; song; brainstem; corollary; bird; vocal

\section{Introduction}

Neural control of complex behavior is often examined in the context of hierarchical models, whereby forebrain structures generate commands that drive other structures, such as the brainstem, which in turn control the peripheral musculature (Kalaska et al., 1997; Suthers and Margoliash, 2002; Ashe et al., 2006). With the recent exception of the motor control of eye movement (Sommer and Wurtz, 2004a,b; Wurtz et al., 2005), little is known about how brainstem nuclei involved in motor output might influence the generation of motor commands by forebrain structures. This is particularly true for vocal motor behavior, where respiratory control is intimately linked to vocal production (Jürgens, 2002; Goller and Cooper, 2004). Despite previous advances in our understanding of brainstem respiratory networks (Ramirez et al., 2004; Feldman and Del Negro, 2006), few attempts have been made to integrate their function into the context of vocal control. Brainstem respiratory areas are often exclusively viewed as output structures, despite robust projections from the brainstem respiratory centers to forebrain vocal control centers in both mammals (Simonyan and Jürgens, 2005) and birds (Reinke and Wild, 1998; Striedter and Vu, 1998).

Received March 19, 2007; revised Jan. 11, 2008; accepted Jan. 24, 2008.

This work was supported by National Institutes of Health Grant R01 DC006102. We thank Jason Wester who contributed to some of the early experiments during his lab rotation, Jessica Cardin for her critical reading of this manuscript, and David Vicario, Josh Gold, Diego Contreras and members of the Schmidt laboratory for reading an earlier version of this manuscript. We also thank Martin Wild for his help in identifying the respiratory brainstem and for his continuous intellectual support.

Correspondence should be addressed to Marc F. Schmidt, Department of Biology, University of Pennsylvania, 312 Leidy Laboratories, 433 South University Avenue, Philadelphia, PA 19104-6018. E-mail: marcschm@sas.upenn.edu. R. C. Ashmore's present address: Department of Neuroscience, University of Pittsburgh, Pittsburgh, PA 15260. D01:10.1523/JNEUROSCI.4547-07.2008

Copyright $\odot 2008$ Society for Neuroscience $\quad$ 0270-6474/08/282613-11\$15.00/0
Vocal production in songbirds is a temporally precise learned behavior that is controlled by a specialized neural circuit, known as the song system (Fig. 1). It contains nuclei in the forebrain, such as HVC (used as a proper name) and its downstream target, the robust nucleus of the arcopallium (RA), which are thought to generate the motor program for song (Vu et al., 1994; Yu and Margoliash, 1996; Hahnloser et al., 2002; Suthers and Margoliash, 2002; Fee et al., 2004; Solis and Perkel, 2005). Output from RA then innervates the brainstem hypoglossal nucleus (nXIIts), which controls syringeal muscles, as well as brainstem vocal respiratory nuclei which, directly [nucleus paraambigualus (PAm) and nucleus retroambigualis (RAm)] or indirectly [the dorsomedial nucleus of the intercollicular complex (DM)], modulate the activity of inspiratory and expiratory motor neurons (Nottebohm et al., 1976; Vicario, 1991; Williams et al., 1992; Wild, 1993; Vates et al., 1997; Reinke and Wild, 1998; Striedter and Vu, 1998; Wild et al., 2000) (Fig. 1). Given the similar organization of respiratory centers in mammals and birds (Douse and Duffin, 1992; Wild, 2004), PAm and RAm, respectively, have been suggested to be homologous to inspiratory and expiratory nuclei of the medullary ventral respiratory group (Wild, 2004).

Anatomical tracing reveals that PAm and DM send bilateral ascending projections to the posterodorsal thalamic nucleus uvaeformis (Uva), a major source of ipsilateral inputs to HVC (Nottebohm et al., 1982; Reinke and Wild, 1998; Striedter and $\mathrm{Vu}, 1998$; Coleman and Vu, 2005). Combined with findings that microstimulation delivered to PAm, but not nXIIts, during singing causes disruptions of song sequencing (Ashmore et al., 2005), these results suggest that PAm can influence song pattern generation, possibly through its ascending thalamic projections. These projections may provide song-related information to vocalcontrol nuclei in both hemispheres and therefore also provide 
interhemispheric temporal coordination (Schmidt, 2003). In this study, we used a combination of microstimulation, stimulation-evoked vocalizations, targeted lesions, and paired recordings in different nuclei of the song control system to define functional brainstem to forebrain pathways that are critical for relaying bottom-up influences from the brainstem to the forebrain.

\section{Materials and Methods}

Animals. Adult male zebra finches (Taeniopygia guttata) ranging from 120 to $500 \mathrm{~d}$ of age were obtained from our breeding colony and from a local supplier. Birds were housed under constant $12 \mathrm{~h}$ light/dark conditions and given food and water ad libitum. All procedures described here were approved by an institutional animal care and use committee at the University of Pennsylvania.

Stimulation and recording. Functional connectivity was established by performing brief microstimulation in one structure and recording extracellularly at one or more contralateral locations under anesthesia. For one experiment, we also recorded from the ipsilateral and contralateral HVC during stimulation of one PAm. Adult male zebra finches were anesthetized with $0.3-0.4 \mathrm{ml}$ of ketamine/xylazine $(40 \mathrm{mg} / \mathrm{kg}$ ketamine and $8 \mathrm{mg} / \mathrm{kg}$ xylazine; Phoenix Pharmaceuticals, Belmont, CA), supplemented with $0.2-0.4 \mathrm{ml}$ of diazepam $(7.5 \mathrm{mg} / \mathrm{kg}$; Abbott Laboratories, Abbott Park, IL).

HVC, RA, PAm, the lateral magnocellular nucleus of the anterior nidopallium (LMAN), and Uva were identified using stereotaxic coordinates combined with electrophysiological recordings to search for characteristic neural activity patterns. For HVC, RA, and LMAN this consisted of spontaneous activity that was considerably higher than surrounding areas and frequently appeared as arrhythmic bursts. RA recording and stimulating sites were specifically targeted to those regions of the nucleus showing the most salient bursting activity. PAm was identified at previously determined stereotaxic coordinates (Reinke and Wild, 1998) in conjunction with recordings of a strong respiratory rhythm in phase with inspiration, as determined by correlated signs of thoracic expansion or recordings of air sac pressure. Uva was identified by the presence of both neural responses to flickering light and somatosensory responses to brushing the feathers. In cases where stimulation was performed in RA, DM, or PAm, the Uva contralateral to these structures was further verified by the presence of evoked responses with a short latency $(<10 \mathrm{~ms})$ after brief trains of stimuli (2-6 biphasic $400 \mu \mathrm{s}, 60 \mu \mathrm{A}$ pulses within 2-6 ms). The medial magnocellular nucleus of the anterior nidopallium (MMAN) was identified by first finding LMAN as described above, and then moving medially (400-700 $\mu \mathrm{m}$ off the midline) and searching for evoked activity from brief stimulation in the contralateral RA or DM. DM was identified using stereotaxic coordinates combined with stimulation to evoke vocalization ( $1 \mathrm{~s}$ of $400 \mu \mathrm{s}, 20 \mu \mathrm{A}$ biphasic pulses at $100 \mathrm{~Hz}$ ) (see below).

Once sites were established, stimulation was delivered to one structure (HVC, RA, DM, or PAm) using a concentric bipolar electrode (FHC, Bowdoin, ME) attached to an isolated pulse stimulator (model 2100; A-M Systems, Carlsborg, CA). Unless otherwise stated, stimuli consisted of 1 biphasic $60 \mu \mathrm{A}$ pulse that was $400 \mu \mathrm{s}$ in duration, or 2 such pulses delivered within $2 \mathrm{~ms}$. We also tested lower current intensities (30-45 $\mu \mathrm{A})$; however, our analysis was performed on data derived from $60 \mu \mathrm{A}$ stimulation. In most cases, these stimuli were delivered every $2 \mathrm{~s}$ for periods ranging from 5 to $10 \mathrm{~min}$. Extracellular activity was recorded with either thin tungsten electrodes ranging from $500 \mathrm{~K} \Omega$ to $2.5 \mathrm{M} \Omega$
(FHC), or with saline-filled glass pipettes. Neural data were filtered between 500 and 10,000 Hz using a two-channel amplifier (Tucker-Davis Technologies, Alachua, FL), and digitally acquired either through a high fidelity sound card (Aardvark Direct Pro) at a sampling rate of $44,100 \mathrm{~Hz}$, or at 20,000 Hz using a four-channel data acquisition device (Micro 1401 MK II; Cambridge Electronic Design, Cambridge, UK) in conjunction with Spike2 software (Cambridge Electronic Design).

Correlation of neural activity between both sides of the brain was established by recording simultaneously in one PAm and in the contralateral RA. Two characteristic types of neurons were identified in PAm: those that showed a clear respiratory rhythm, and those that produced arrhythmic bursts that were not correlated to the respiratory pattern. To be assured that these bursting neurons were in PAm, we only recorded from sites where both bursts and one respiratory unit were present, or where bursts were located between respiratory units recorded both above and below within $50 \mu \mathrm{m}$ on the same electrode penetration. In two cases, electrode placement was verified by performing small lesions in PAm at the recording sites, and verifying the lesion locations by frozen section histology (see below).

Respiratory monitoring. For detailed analysis of respiratory cycle and related neural activity, air sac pressure recording was performed as previously described (Franz and Goller, 2002; Ashmore et al., 2005). Briefly, under anesthesia, a thin pressure cannula was inserted into the abdominal air sac on the left or right side just below the lowest rib. This cannula was connected to a small pressure transducer (FHM-02PGR-02; Fujikura, Tokyo, Japan) that was powered by a custom-built power source (provided by Brent Cooper). For other experiments, thoracic expansion was monitored less invasively with a plastic air-filled cushion placed between the bird's chest and a jacket that held the cushion in place, and this device was attached via a tube to the pressure transducer. In both cases, the signal was amplified and bandpass filtered between $0.1 \mathrm{~Hz}$ and $100 \mathrm{~Hz}$ using a Brownlee (San Jose, CA) amplifier (model 440), and acquired at $20,000 \mathrm{~Hz}$ using a four-channel data acquisition device (Micro 1401 MK II; Cambridge Electronic Design) in conjunction with Spike2 software (Cambridge Electronic Design). 
Lesions. Lesion targets (Uva and MMAN) were first located as described above, and mapped out using a tungsten electrode (FHC). For both Uva and MMAN, between one and seven overlapping lesions were performed, and when contralateral RA, PAm, or DM stimulation was used, the lesions were centered around the site of greatest evoked activity from stimulation at these sites. To perform the lesions, $15 \mu \mathrm{A}$ of direct current was delivered through the recording electrode for $60 \mathrm{~s}$ using a Midgard Current Source (Stoelting). All lesions were verified by frozen section histology (see below).

Lesions of Uva ranged from 25 to $100 \%$, with more than half of these covering $>75 \%$ of the nucleus. All lesions of MMAN were incomplete, ranging from $\sim 25$ to $75 \%$ of the area. In some cases with both MMAN and Uva, lesions encompassed some of the surrounding tissue, and these were included in subsequent analysis. Lesions that did not impinge on their intended targets had no effect on neural activity and were not included for analysis.

Evoking vocalization under anesthesia. Call-like vocalizations were elicited with DM stimulation (EVOCs) as described previously (Vicario and Simpson, 1995). A monopolar or concentric bipolar electrode (FHC) was inserted into one DM, and long stimulus trains were delivered using an isolated pulse stimulator (model 2100; A-M Systems). Individual pulses were $400 \mu$ s long, ranging in amplitude from 15 to $40 \mu \mathrm{A}$. The frequency of stimulation ranged from 10 to $20 \mathrm{~Hz}$, and the train duration ranged from 300 to $1000 \mathrm{~ms}$ for a total of 3-20 stimuli. For each bird, the parameters were set to maximize the probability and number of vocalizations that could be elicited in the time period following stimulus offset. In other words, we sought to evoke vocalizations that started after or extended beyond the duration of the stimulation, to isolate vocal-related neural activity from the stimulus artifacts. This ability to elicit such vocalizations was also critical because it allowed the ability to dissociate stimulus-driven neural responses (direct trans-synaptic activation) from those that were linked to vocal production. Although vocalizations could be evoked reliably during the stimulation period, production of vocalizations that were completely separated from the stimulus were more difficult and were obtained reliably in only two birds. Therefore we included for analysis all vocalizations that extended to at least $100 \mathrm{~ms}$ beyond the end of stimulation, regardless of whether these vocalizations began during or after stimulation.

Analysis. Poststimulus time histograms (PSTHs) for stimulationevoked activity were constructed by aligning data from the recording electrodes to the time of stimulation. A raster was computed using a simple thresholding algorithm that identified neural events (spikes or peaks, i.e., changes from positive to negative slope) that occurred within an absolute threshold range. This same procedure was applied to recordings with either single or multiunit data. Unless otherwise noted, typical PSTHs were generated from the activity collected from a total of 100 stimuli (delivered at $0.5 \mathrm{~Hz}$ ), which was then compiled into $5 \mathrm{~ms}$ bins. To calculate peak-to-baseline ratios, we took the maximum value of the first 20 bins ( $100 \mathrm{~ms}$ ) after stimulation and divided it by the mean of all bins in the $100 \mathrm{~ms}$ preceding stimulation. As a control, we also calculated a peak-to-baseline ratio using this same baseline period and dividing its mean by the maximum value within this baseline window. Values are stated in the text as the mean \pm SD.

Latency for a given block of stimuli was determined by summing the neural traces after all stimuli and smoothing with a $1 \mathrm{~ms}$ wide Gaussian filter. The time from the beginning of stimulation to the peak of this smoothed trace was used as the "time to peak" measure, and "time to half peak" was calculated as the latency from the beginning of stimulation to reach half of the peak value.

To look for spontaneous activity in PAm neurons that showed a relationship to contralateral RA activity, we constructed perievent time histograms (PETHs) of RA activity aligned to PAm activity. To construct these histograms, multiunit or single-unit recordings from RA were aligned to identified events in the single or multiunit PAm recordings. Because we were looking specifically for bursts of activity in nonrespiratory neurons within PAm, these burst events were identified by an algorithm that computed the rate at which neural events (peaks resulting from the firing of one or more neurons) occurred within a threshold range, and selected only those times where the rate was higher than a chosen threshold $(50-150 \mathrm{~Hz})$. After each burst identification, the algorithm skipped forward in the PAm trace $40 \mathrm{~ms}$ to avoid counting the same burst multiple times. Subsequent manual inspection was performed to verify the accuracy of the algorithm, specifically to verify that the algorithm identified $>90 \%$ of all bursts, that the onset was correctly identified, and that neither respiratory activity nor noise were erroneously counted. RA activity was then aligned to the onset of the PAm burst (the time of the first neural event in PAm identified by the algorithm) for each burst identified. Activity rasters for RA were then constructed as described above, and the events were compiled in $10 \mathrm{~ms}$ bins.

To assess the degree of spontaneous burst coincidence in RA and PAm, we computed the peak-to-baseline ratio for RA activity aligned to each PAm burst (as described above). Peaks in the histogram were drawn from only the first $50 \mathrm{~ms}$ of activity that followed PAm burst onset because our results from trans-synaptic activation of RA from PAm stimulation showed responses that peaked and decayed within this time frame. For comparison, we also performed this same analysis for the $50 \mathrm{~ms}$ preceding PAm burst onset. As a baseline for RA activity in these experiments, the mean baseline values were obtained by randomly drawing 100 periods (50 ms long) of RA activity from the same recording and computing a histogram of expected RA activity. Control values were generated by using this same baseline, compared with the peaks generated from these randomly selected windows (the peaks of the baseline histogram). To ensure that peaks of RA activity aligned to PAm burst onsets were not simply caused by periodic changes in RA or PAm activity, we measured the autocorrelation of PAm and RA activity at each site and verified an absence of peaks outside of the zero time lag vicinity.

Vector strength for respiratory and RA neurons was calculated from single-unit recordings by first calculating the angle in the respiratory cycle at which each spike occurred. To determine the angle, an $80 \mathrm{~s}$ trace of simultaneously recorded air sac pressure (smoothed with a $1 \mathrm{~ms}$ wide Gaussian filter) was separated into individual cycles, with the start of each cycle defined as the time when the pressure trace crossed the ambient threshold from positive (expiration) to negative (inspiration). Each respiratory cycle, and the accompanying spike times for RA and respiratory neurons, was then time warped to match the mean respiratory cycle length. Neural spikes times were then compiled into rasters, and vector strength was computed using a standard algorithm (Goldberg and Brown, 1969). For each unit recorded, control values were generated by shuffling the spike times in each cycle, computing a new vector strength, and repeating this process 100 times to generate a mean and SD. The polar plot shown in Figure $5 B$ was generated from a neural spike raster from one PAm respiratory and one RA unit, compiled into histograms of 36 bins, and then plotted in polar coordinates.

To compute neural activity during EVOCs, each neural recording (in PAm or HVC) was aligned to the offset of the stimulus train, and the timing of neural events was determined using a simple thresholding algorithm as described above. PSTHs shown in Figure 8 reflect all neural activity aligned to stimulus offset, with the stimulation period removed. Analysis for Figure 8,C and $D$, included only neural activity occurring during vocalization. Periods of vocalization were determined by rectifying the audio trace, smoothing (convolving) with a $10 \mathrm{~ms}$ wide Gaussian filter, and applying a threshold to the result. Neural events occurring during the vocalization period were summed, and then normalized by the vocalization duration to compute the activity rate (the number of neural events per second) for each EVOC. Because we were interested in activity associated with the ongoing vocalization, and wanted to exclude immediate activity resulting directly from trans-synaptic activation of HVC or PAm neurons after stimulation, we excluded from analysis the first $50 \mathrm{~ms}$ after the stimulus train offset. For baseline measurements, we used spontaneous activity levels measured in the period immediately before each stimulation. Data were gathered from five PAm sites in four birds, and from four HVC sites in three birds, and the number of stimulus/vocalization recordings ranged from 20 to 66 for each of the sites. All HVC recordings were obtained from sites that were recorded simultaneously with PAm. For each site, the mean vocalization-related activity rate was compared with the mean baseline activity rate, and significance was determined by paired $t$ test. To estimate activity across the population, the compiled grand means of neural rates for all PAm and all HVC 
recording sites were then compared with their respective baseline grand means, and significance was again determined by paired $t$ test.

All analysis was performed using custom scripts written in Matlab (The MathWorks, Natick, MA).

Histology. Birds were deeply anesthetized with $0.1 \mathrm{ml}$ of $50 \mathrm{mg} / \mathrm{ml} \mathrm{Nembutal}$ (Abbott Laboratories) and perfused with $0.9 \%$ saline and $4 \%$ paraformaldehyde. Brains were cryoprotected in $30 \%$ sucrose and sectioned at $40 \mu \mathrm{m}$ on a freezing microtome. All sections were cut coronally and stained with cresyl violet.

\section{Results}

Stimulation in the brainstem vocalrespiratory network activates the contralateral forebrain song system

We examined the effects of stimulation in the inspiratory-related nucleus PAm, which receives input from the ipsilateral RA and projects bilaterally to Uva. We recorded contralateral RA activity during 100 consecutive stimuli (spaced $2 \mathrm{~s}$ apart) delivered to PAm in 4 birds. With as few as one or two biphasic pulses (400 $\mu$ s, 30 or $60 \mu \mathrm{A}$ ), we found that stimulation in PAm produced consistent, robust responses in the contralateral RA (Fig. 2A), even though these nuclei are separated by at least three synapses. With $60 \mu \mathrm{A}$ stimulation, responses were seen after $>90 \%$ of stimulations. Responses typically appeared as an increase in multiunit firing rates followed by temporary (50-100 ms) suppression below baseline activity levels. When plotted on a PSTH, peak responses ranged from 4.8-29.9 times the magnitude of the baseline activity (peak-to-baseline ratio) (for details, see Materials and Methods) with a mean peak-to-baseline ratio of $15.5 \pm 10.5$ ( $n=4$ birds). This was significant when compared with control peak-to-baseline measurements $(1.4 \pm 0.1 ; p<$ 0.05 , paired $t$ test; $n=4)$. To estimate the response latency, we calculated the time from stimulus onset to the response half-peak value (time-to-half-peak) for the compiled data from the 100 trials for each bird. For PAm stimulation, the latency to half peak in RA was $12.7 \pm 2.4 \mathrm{~ms}$ (Fig. $2 C)(n=4$ birds), consistent with a propagation delay across three synapses. In one bird, stimulation in PAm produced a biphasic response in the PSTH compiled over 100 trials, a phenomenon not seen in other birds given PAm stimulation, or from stimulation in other areas. This second peak occurred with a latency of $35.3 \mathrm{~ms}$ (measured from the time of stimulation).

Stimulation in DM, which like PAm receives input from the ipsilateral RA and projects bilaterally to Uva, also consistently evoked activity in the contralateral forebrain song control nuclei (Fig. 2 B). Evoked responses in RA ranged from 3.0-8.7 times the magnitude of the baseline activity with a mean peak-to-baseline ratio of $5.4 \pm 1.7$ ( $n=9$ birds). Responses were significant when compared with control peak-to-baseline measurements (1.3 \pm $0.1 ; p<0.0001$, paired $t$ test; $n=9)$. Stimulation outside of DM (as revealed by failure to evoke vocalization with 1-s trains of stimuli) always failed to evoke a response in RA ( $n=9$ birds). For
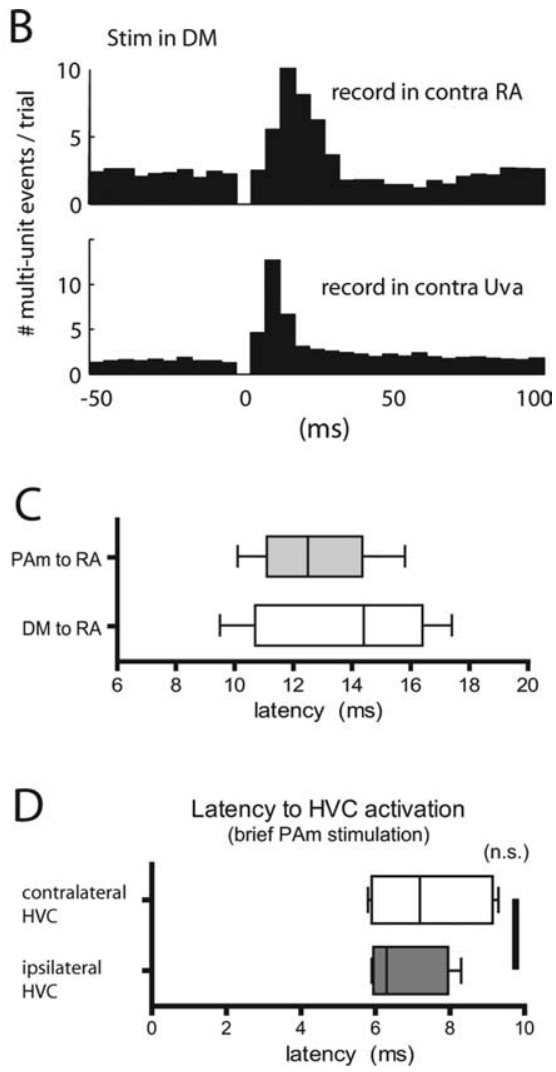

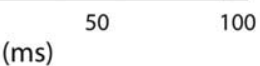

Figure 2. Stimulation of the respiratory brainstem activates contralateral Uva, RA, and HVC. $\boldsymbol{A}$, Effect of PAm stimulation on activity in contralateral Uva and RA. Middle, PSTH of RA activity after 100 stimulations in PAm of which four sample multiunit above the histogram. Stimulation occurred at time 0 and the stimulus artifact has been removed from the neura traces and the PSTH. The bottom panel represents the PSTH of activity recorded simultaneously in Uva contralateral to the PAm site. In this example, stimulation consisted of two $400 \mu$ s wide, $60 \mu \mathrm{A}$ biphasic pulses within $2 \mathrm{~ms}$. $B$, Effect of Dh the left DM. C, Latency for contralateral RA responses after stimulation in PAm and DM. Latency was measured from the sum of the to reach half of the peak value of this summed trace. $\boldsymbol{D}$, Latency to activate both the contralateral (white) and ipsilateral (gray) HVC after stimulation in PAm. Latency distribution for $\boldsymbol{C}$ and $\boldsymbol{D}$ is shown as a box-and-whisker plot.

DM stimulation, the mean time to half peak was $13.4 \pm 2.8 \mathrm{~ms}$ (Fig. $2 C)(n=9$ birds).

To investigate the path of propagation from one hemisphere to the other, we also recorded from the contralateral Uva during stimulation in PAm or DM in a subset of birds while recording simultaneously in the contralateral RA. Stimulation in PAm evoked activity in the contralateral Uva with a mean peak-tobaseline ratio of $9.5 \pm 7.2(p<0.05$ compared with control ratio of $1.6 \pm 0.2$; Kruskal-Wallis ANOVA used because of small nonnormal sample; $n=4)$. Stimulation in DM evoked a mean response of $5.9 \pm 2.4$ ( $p<0.0005$, paired $t$ test; $n=8$ birds $)$. Although the peak of the response was clearly evident after stimulation in PAm or DM, latencies for evoked activity (time to half peak) in the contralateral Uva were difficult to estimate because the onset (and the time of the half-peak value) of the Uva response often fell within the range of the stimulus artifact $(<3 \mathrm{~ms})$.

We next investigated whether Uva is critical for the ability of brainstem stimulation in PAm and DM to evoke activity in the contralateral RA. After collecting baseline responses in RA and Uva after stimulations in PAm or DM, we then made electrolytic lesions targeted around the site of the greatest evoked response in Uva. Lesions of Uva completely abolished PAm-evoked responses in RA (Fig. $3 \mathrm{~A}$ ). The mean postlesion peak-to-baseline 

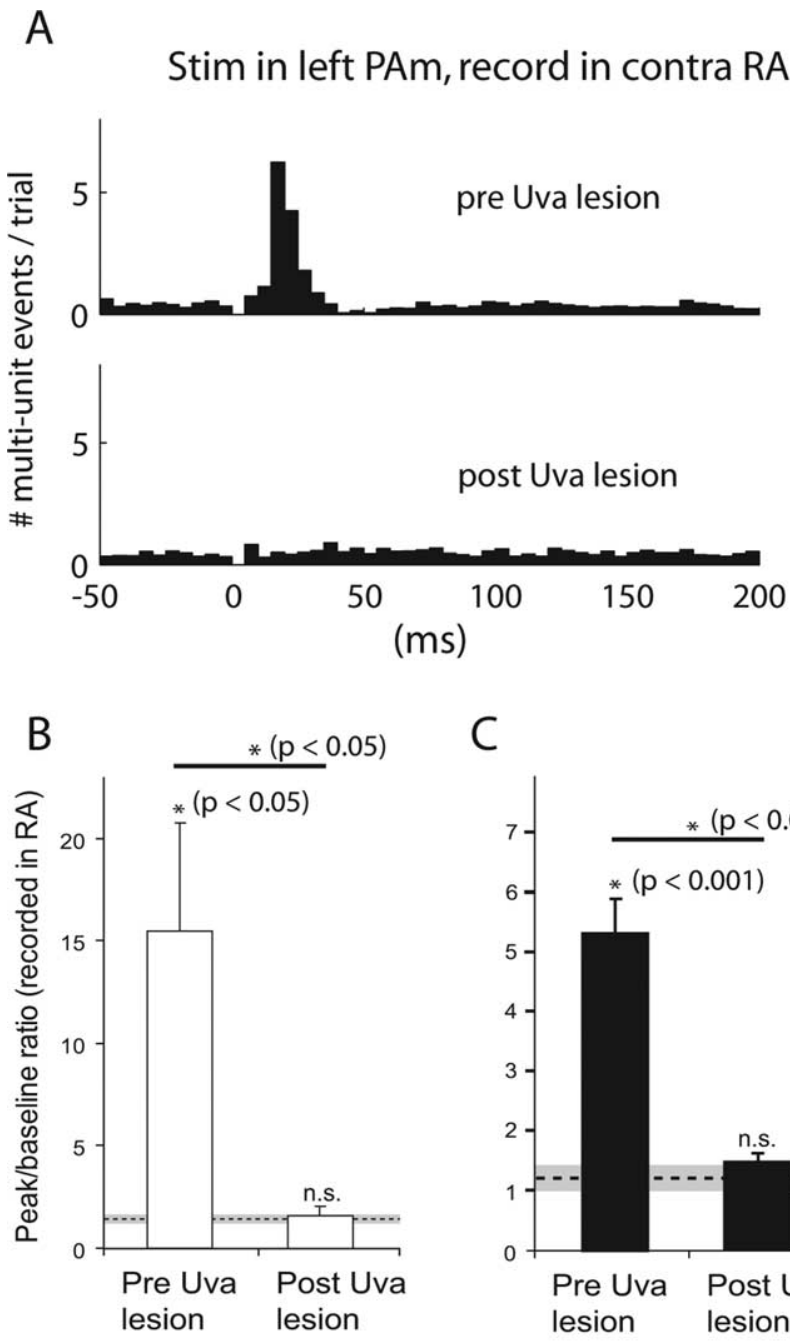

PAm

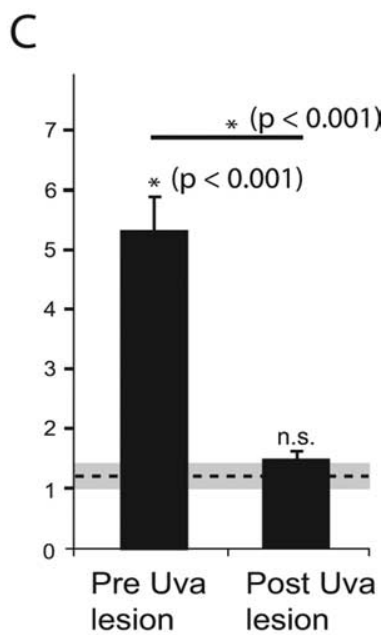

DM

Figure 3. Lesions of Uva block brainstem to forebrain signal propagation. $A$, PSTH showing activity after stimulation in the left PAm recorded in the contralateral RA, before and after electrolytic lesions of the right Uva. B, Comparison of evoked responses before and after Uva lesion. Measurements of evoked responses in RA (contralateral to stimulation) are shown for birds receiving stimulation in PAm $(n=4)$. The $y$-axis values indicate the mean peak-tobaseline ratio for each group (see Materials and Methods). The dotted line represents the mean control peak-to-baseline value calculated before Uva lesions $(n=4)$, and the gray area represents two SDs from this mean. Error bars represent SEM. Asterisks indicate significance for the group by one-tailed paired $t$ tests, wherein each bird was paired with either its own control peak-to-baseline value, or paired before and after Uva lesions. C, Comparison of evoked responses from stimulation in DM $(n=9)$ before and after Uva lesions.

value $(1.6 \pm 0.8)$ was significantly lower than the prelesion value $(15.5 \pm 10.5, p<0.05$, paired $t$ test, $n=4)$, and not significantly different from control $[1.6 \pm 0.2$, not significant (NS); paired $t$ test; $n=4$ ] (Fig. $3 B$ ). Similar results were obtained for DM stimulation. Mean peak-to-baseline ratio decreased from $5.4 \pm$ $1.7(p<0.001$; paired $t$ test; $n=9)$ to $1.5 \pm 0.1$ after the lesion, which was not significantly different from control values $(1.3 \pm$ 0.1 , NS; paired $t$ test; $n=9$ ) (Fig. $3 C$ ).

One possible role of the ascending bilateral projections of PAm might be to serve as synchronizing signals for the forebrain song system nuclei, thus contributing to interhemispheric coordination. A prediction of this hypothesis is that signals from one PAm would influence activity in both HVCs with a similar time course. To test this, we performed stimulation in one PAm and
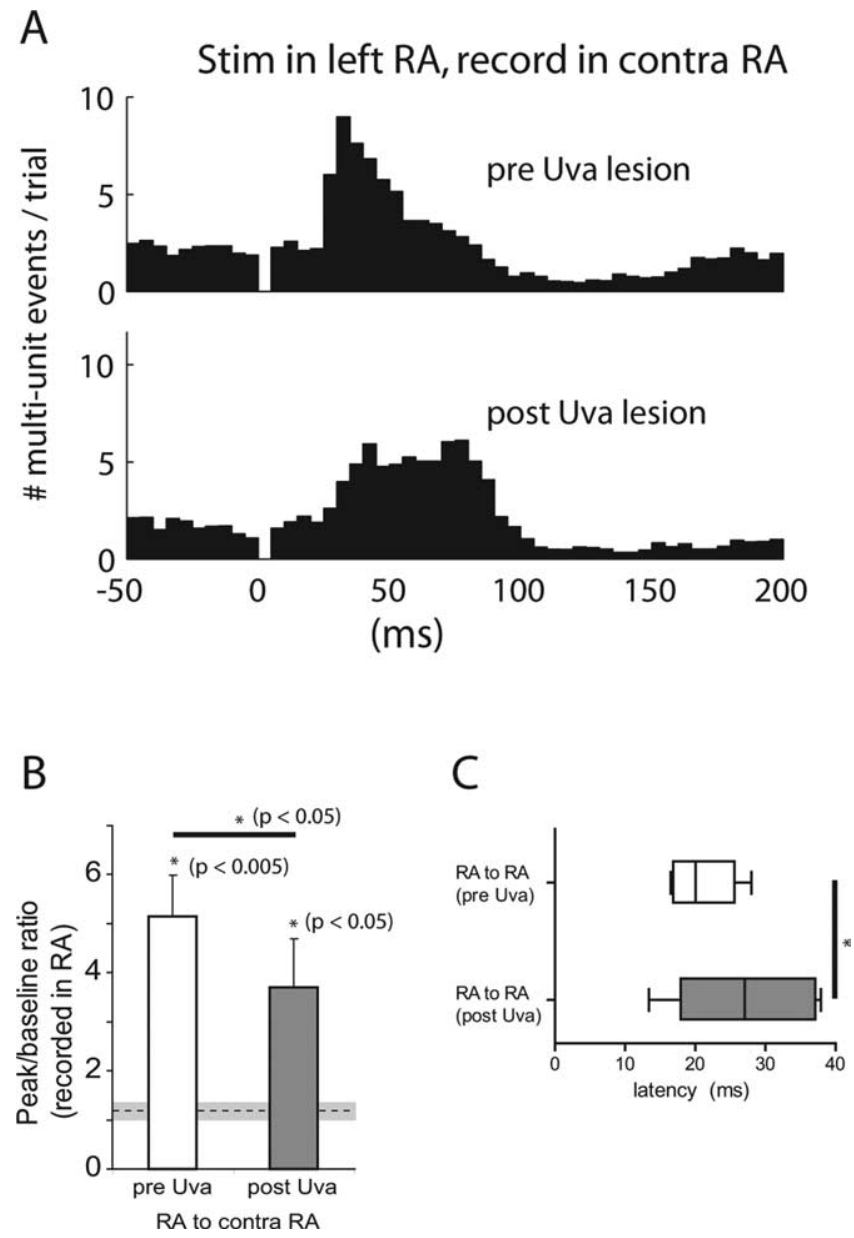

Figure 4. Cross-hemisphere activation is partially dependent on the Uva-mediated relay pathway. A, PSTHs of right RA activity after stimulation in the left RA, before (top) and after (bottom) lesion of the intermediate right Uva. In this example, the lesion attenuates the RA response and causes a shift in peak latency. $\boldsymbol{B}$, Mean peak-to-baseline ratios for six birds with stimulation delivered in one RA and recordings made in the contralateral RA. Mean control value \pm two SDs is shown as a dotted line surrounded by a gray box, and was calculated from randomly sampled RA traces before the Uva lesion. Asterisks represent significance by $t$ tests. Error bars indicate SE. C, Latency to the evoked response from RA stimulation, recorded in the contralateral RA, measured as time to half peak.

simultaneously recorded multiunit activity in both HVCs. PAm stimulation evoked robust neural responses in both ipsilateral and contralateral HVC ( $n=5$ stimulation sites in 4 birds). The latency to response in the ipsilateral HVC was $6.8 \pm 1.0 \mathrm{~ms}$, which was not significantly different from the latency $(7.5 \pm 1.5 \mathrm{~ms})$ observed in the contralateral HVC (Fig. $2 D)(p=0.46$, paired $t$ test; $n=5$ stimulation sites). The difference between the variances of the two latencies was also not significant ( $p=0.43, F$ test).

\section{Stimulation of forebrain song control nuclei in one}

hemisphere evokes activity in the contralateral hemisphere The results described above indicate that stimulation in the vocal-respiratory brainstem evokes activity in song control nuclei of the contralateral forebrain. These data suggest that stimulation of forebrain areas in one hemisphere should elicit activity in the contralateral hemisphere via the brainstem-thalamus pathway. We therefore stimulated in one RA and recorded contralateral RA activity. To allow for direct comparison with the effect of brainstem stimulation, we recorded activity at the same contralateral 
RA site during stimulation of both RA and DM in five of six of these experiments. Stimulation of RA with 100 consecutive stimuli evoked responses in the contralateral RA that were comparable in size to those elicited by stimulation in PAm or DM, even though RA is separated by at least four synapses from its contralateral counterpart (Fig. 4A). The mean peak-tobaseline ratio of $5.2 \pm 2.0$ was significantly larger than control $(1.2 \pm 0.1 ; p<0.005$, paired $t$ test; $n=6$ birds). Stimulation immediately outside of RA had no effect ( $n=$ 6 birds). Stimulation in RA also evoked responses in the contralateral Uva with a mean peak-to-baseline ratio of $3.5 \pm 1.4$ ( $p<0.05$, paired $t$ test; $n=4$ birds). Mean time to half peak latency for contralateral RA responses after RA stimulation was $20.9 \pm 4.5 \mathrm{~ms}$, which was significantly longer than the latency for DM stimulation $(13.4 \pm 2.8 \mathrm{~ms} ; p<0.01, t$ test; $n=6 \mathrm{RA}$ and $9 \mathrm{DM}$ ) (Fig. $4 C$ ). As predicted by the synaptic distance, the latency to contralateral Uva activation after RA stimulation $(9.7 \pm 2.4 \mathrm{~ms})$ was shorter than the latency to evoke activity in the contralateral RA ( $p<0.0005$, $t$ test; $n=6$ birds for RA and 4 birds for Uva).

The same lesions of Uva that were sufficient to abolish the DM stimulation evoked response in the contralateral RA (Fig. 3C) attenuated, but did not completely eliminate, the response caused by stimulation in RA (Fig. 4B). Mean peak-tobaseline ratio $(3.7 \pm 2.4)$ was significantly smaller than prelesion values $(5.2 \pm 2.0$; $p<0.05$, paired $t$ test; $n=6$ birds), but was still significantly higher than the control values $(1.3 \pm 0.1 ; p<0.05, t$ test; $n=6$ birds $)$. In addition to decreasing response strength, Uva lesions also increased the response latency from $20.9 \pm 4.5$ to $27.1 \pm 9.1 \mathrm{~ms}(p<0.05$, paired $t$ test; $n=6$ ) (Fig. $4 C$ ).

One possible explanation for the remaining response is that RA, unlike DM and PAm, sends projections to song nuclei in the contralateral forebrain along two anatomically identified pathways. One pathway leads through the vocal-respiratory network (including DM and PAm). The other pathway projects to the contralateral HVC and RA via the dorsomedial posterior nucleus of the thalamus (DMP) and the forebrain nucleus MMAN (Fig. 1, green pathway). To test the contribution of this second pathway, we stimulated in RA and measured evoked activity in the contralateral MMAN in 5 birds that had previously received lesions of Uva. Stimulation in RA reliably evoked responses in MMAN (supplemental Fig. $1 A$, available at www.jneurosci.org as supplemental material), with a peak-to-baseline ratio of $14.5 \pm 12.7$, and a latency of $14.7 \pm 4.2 \mathrm{~ms}(n=5)$. Activity recorded simultaneously in the contralateral RA revealed a mean evoked response of $5.3 \pm 0.9$, and a latency of $23.7 \pm 7.7 \mathrm{~ms}(n=5$ birds $)$ (supplemental Fig. $1 B$, available at www.jneurosci.org as supplemental material).

After verifying evoked activity in the contralateral MMAN and RA of this subset of Uva-lesioned birds, we then performed

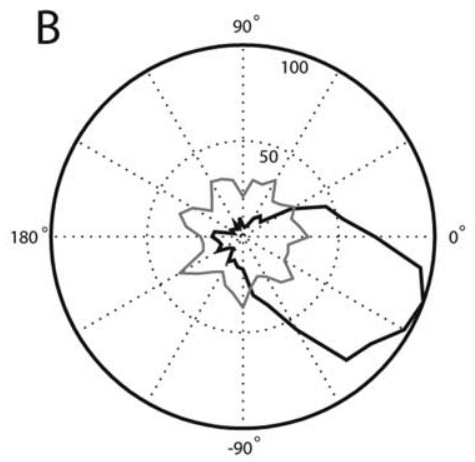

D

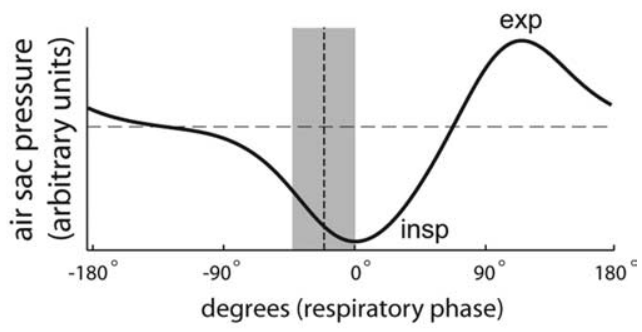

Inspiratory

$-200 \mathrm{~ms}$

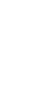
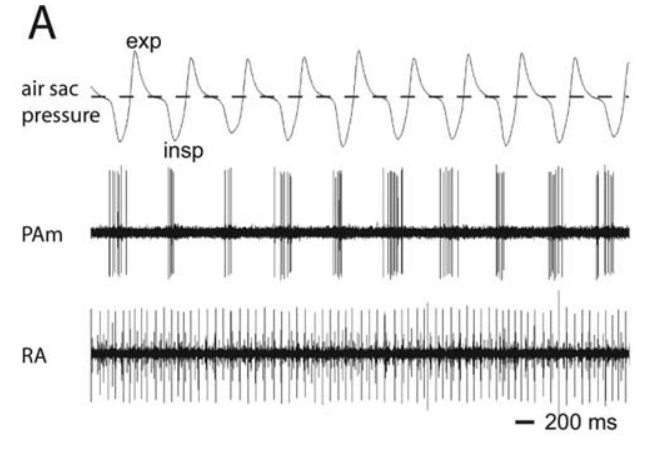

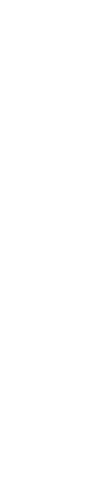

Figure 5. Characterization of respiratory phase relationship in RA and PAm respiratory neurons. $A$, Simultaneous recordings of air sac pressure (top), single-unit activity in left PAm (middle), and unit activity in contralateral RA (bottom). Expiration (exp) is indicated as a positive deflection in the air sac pressure trace, whereas inspiration (insp) is indicated as negative deflections. The gray. In this figure, $0^{\circ}$ is the approximate peak of the inspiratory phase. $C$, Mean vector strength for eight RA (left) and eight PAm inspiratory (right) neurons, compared with the mean of the vector strengths calculated by shuffling the spike times of each one respiratory cycle. The mean phase-relative timing of eight PAm inspiratory neurons is overlaid on the trace as a vertical dotted line. The surrounding gray box represents two SDs from this mean.

partial unilateral lesions of MMAN at the site of the greatest evoked response. We found that these lesions further attenuated the RA to RA response to $3.3 \pm 1.4$ from $5.3 \pm 0.9$ immediately before lesion in these 5 birds ( $p<0.05$, paired $t$ test, $n=5$ ). MMAN lesions did not completely eliminate the evoked activity as responses were still higher than the control value $(1.3 \pm 0.2$; $p<0.05, t$ test; $n=5$ ) (supplemental Fig. $1 B$, available at www. jneurosci.org as supplemental material), possibly reflecting the fact that the MMAN lesions were estimated to be incomplete (as a consequence of the diffuse nature of the structure). Latency for the evoked response did not significantly increase $(25.9 \pm 10.1$ $\mathrm{ms})$. Because MMAN lesions in four of these birds were performed 1 week after lesions of Uva, we were concerned that neural plasticity during this intervening period might impact our results. We therefore performed combined Uva and MMAN lesions within the same recording session while stimulating both DM and RA and recording in the contralateral RA ( $n=1$ bird). Complete results for this bird are shown in supplemental Figure $1 C$ (available at www.jneurosci.org as supplemental material) and reveal the same trends as the whole population.

\section{RA firing is not strongly correlated with respiratory phase}

PAm is part of a network of nuclei responsible for generating the normal respiratory rhythm, and as such, the majority of neurons 


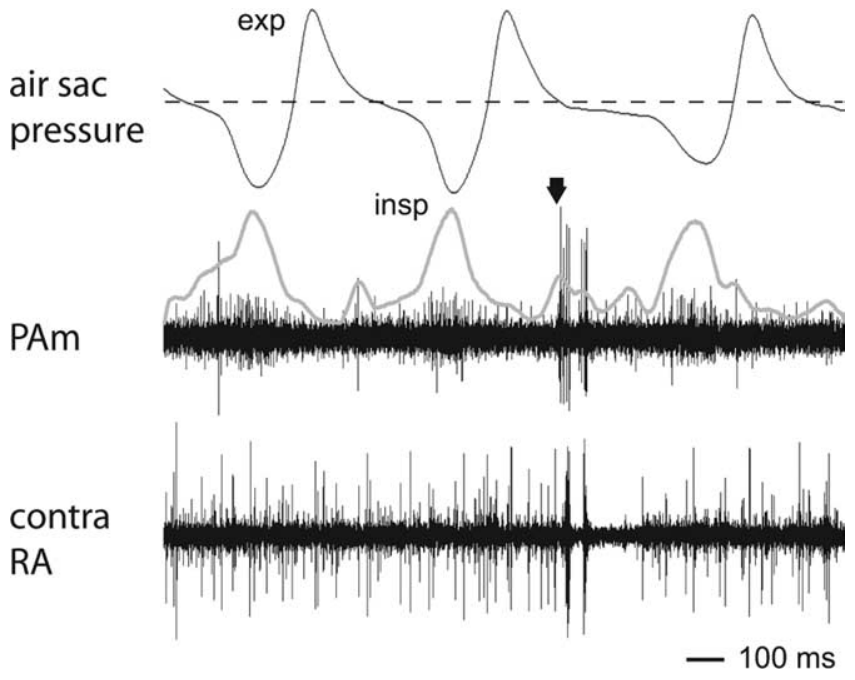

Figure 6. Identification of nonrespiratory bursting neurons in PAm that are spatially near inspiratory neurons. Multiple unit activity was recorded in PAm (center trace) simultaneously with activity in the contralateral RA (bottom trace) and with air sac pressure (top trace). The arrow indicates the time of simultaneous bursting in RA and the nonrespiratory PAm unit. Also visible in the background of the PAm electrode are units that fire in phase with inspiration. The thick gray line is a rectified and smoothed version of the PAm trace after the burst spikes have been removed, highlighting the respiratory rhythm of this background activity. In this example, the respiratory cycle was longer when a burst occurred than when the PAm unit remained silent. Changes in respiratory cycle length were occasionally observed during bursts in PAm and/or RA; however, this phenomenon was not consistently seen. exp; Expiration; insp, inspiration.

in PAm are thought to fire in phase with inspiration (Reinke and Wild, 1998). A typical phase-locked inspiratory neuron in PAm is shown in Figure $5 A$ (middle trace). In this example, recordings were obtained simultaneously with air sac pressure (Fig. $5 A$, top trace) to compare neural activity with the breathing rhythm (Fig. $5 A$ ). We found that different PAm neurons had different phase relationships with respiration, and some fired at the onset of the inspiratory phase, whereas others (Figs. 5, 6) were delayed with respect to inspiratory onset. In addition, different neurons also had different duty cycle lengths, similar to respiratory neurons in mammalian systems. In contrast to PAm neurons, recordings in Uva (data not shown) or RA (Fig. 5A) never revealed any obvious rhythmic activity despite the ability of stimulation in PAm to evoke robust responses in RA in an Uva-dependent manner. Instead, RA neurons showed tonic firing, punctuated by occasional bursts that were frequently followed by silent pauses, none of which were modulated by the respiratory cycle (Fig. $5 A$, bottom trace).

To quantify these observations, we recorded from eight inspiratory-related units in PAm and eight RA units in four birds, and examined their firing relative to simultaneously recorded air sac pressure. We used the air sac pressure recordings to determine the respiratory phase (expiration versus inspiration), and we compiled activity for the neurons relative to this phase across multiple cycles. As expected, these PAm neurons showed a strong phase relationship to respiration, firing predominantly during the inspiratory part of the cycle (Fig. $5 B, D$ ). We computed vector strength for these neurons and compared these values to a set of vector strengths computed from time-shuffled controls for each neuron. The vector strengths of all eight PAm neurons were significant compared with their own controls $(p<0.001$, z test; $n=$ $8)$. Across the population, the mean vector strength was $0.67 \pm$ 0.15 , which was highly significant compared with the mean vector strength of the shuffled controls $(0.03 \pm 0.02 ; p<0.00001$, paired $t$ test; $n=8$ ) (Fig. $5 C$ ). By comparison, only one of the eight RA units demonstrated significant vector strength relative to its own shuffled control $(0.18$ vs $0.03 \pm 0.02$ for the shuffled set; $p<0.001, z$ test; $n=1$ of 8 ). As a population, the mean vector strength of the RA units was $0.04 \pm 0.06$, which was not significantly different from the mean of the shuffled controls $(0.03 \pm$ 0.01, NS; $n=8$ ) (Fig. 5C).

\section{Identification of a novel nonrespiratory bursting neuron type in PAm}

The near absence of a respiratory rhythm in either Uva or RA suggests the possibility that PAm contains two distinct populations of neurons: Neurons that are phase locked to the respiratory rhythm, and Uva-projecting neurons activated by stimulation in PAm that are not locked to the respiratory pattern. To investigate this possibility, we searched for neurons in PAm whose firing pattern was correlated with contralateral RA activity, but not with the respiratory rhythm. In each of 12 birds tested, we identified a population of neurons concentrated in the dorsal half of PAm that had low overall spontaneous firing rates $(0.8 \pm 0.4$ spikes/s; $n=8$ isolated units), and that often fired in arrhythmic bursts (within-burst firing rate $128.0 \pm 42.3$ spikes/s; $n=8$ ). The periods of highest activity were typified by multiple closely spaced bursts that were unrelated to the respiratory pattern (Figs. 6, 7). These nonrespiratory PAm neurons were located in close spatial proximity to respiratory neurons, as exemplified by the many cases where we were able to record simultaneously from both types of neurons on the same electrode (Fig. 6). In other cases, these units were found nested between respiratory units both above and below within $50 \mu \mathrm{m}$ on the same electrode penetration.

Simultaneous recording from nonrespiratory bursting neurons in PAm and neurons in the contralateral RA revealed that the PAm bursts were generally correlated with bursting activity in RA (Figs. 6, 7). Specifically, many bursts in the nonrespiratory PAm neurons were seen at approximately the same time as bursting in the contralateral RA neurons. To measure possible correlations between these different cell types, we constructed PETHs of activity in RA, aligned to bursts in this subset of PAm neurons (see Materials and Methods for details). As shown by the peaks surrounding the alignment time $(t=0 \mathrm{~ms})$, activity in RA was significantly elevated during burst periods in the nonrespiratory PAm neurons (Fig. $7 B$, top), compared with shuffled controls (Fig. $7 B$, bottom). In contrast, alignment of RA activity to the first spike of each burst in PAm respiratory neurons failed to show any elevated activity, providing further support for a lack of respiratory-patterned activity in RA (Fig. 7D).

To quantify the degree to which firing in nonrespiratory PAm neurons was coincident with firing in RA, we calculated the peak value of the PETH in a $50 \mathrm{~ms}$ window following the zero time point (the PAm burst time), and computed a peak-to-baseline ratio using a baseline drawn from randomly selected $50 \mathrm{~ms}$ periods of the recorded RA activity (for details, see Materials and Methods). The mean peak-to-baseline ratio for RA activity during the $50 \mathrm{~ms}$ after alignment to nonrespiratory PAm neuron burst onset was $4.6 \pm 2.1$ and was significantly different from activity levels obtained by randomly sampling RA activity (1.6 \pm $0.2 ; p<0.05$, one-tailed paired $t$ test; $n=5$ ) in this population of neurons (Fig. 7D). The mean peak-to-baseline ratio for the same RA activity aligned to respiratory PAm neurons was $1.6 \pm 0.2$ and was no different from the control value (NS, one-tailed paired $t$ test; $n=5)$.

To investigate whether the pathway from PAm to the con- 
tralateral RA contributes to the observed correlation between these two structures, we recorded simultaneous neural activity from nonrespiratory neurons in PAm and the contralateral RA both before and after lesions of Uva in 5 birds. Uva lesions caused a significant decrease in the mean peak-tobaseline ratio for the $50 \mathrm{~ms}$ following burst alignment (prelesion, $3.9 \pm 1.7$; postlesion, $2.3 \pm 0.4 ; p<0.05$, one-tailed paired $t$ test; $n=5$ ). The decreased post-Uva lesion value remained nevertheless significantly higher than the control value derived from prelesion shuffled RA activity $(1.5 \pm 0.4$; $p<0.05$, one-tailed paired $t$ test; $n=5$ ).

We then examined RA activity for the 50 ms immediately preceding PAm bursts, and found that activity during this period was elevated relative to shuffled RA activity, with a peak/baseline ratio of $3.0 \pm 0.9(p<$ 0.05 , one-tailed paired $t$ test; $n=5)$. Interestingly, contrary to the $50 \mathrm{~ms}$ after PAm bursts, Uva lesions reduced but did not significantly attenuate peak/baseline values $(2.4 \pm 1.1 ; p=0.2$, one-tailed paired $t$ test; $n=5$ ) during the $50 \mathrm{~ms}$ that preceded the PAm burst. Together, these results suggest that Uva lesions attenuate some of the correlation between nonrespiratory PAm neurons and the contralateral RA, but do not eliminate it, particularly for RA activity that precedes bursts in PAm.

\section{Nonrespiratory PAm neurons are active during vocalizations evoked under anesthesia}

Because nonrespiratory bursting PAm neurons are correlated with activity in the song system, we hypothesized that these neurons form part of the song motor circuit. One prediction of this hypothesis is that these neurons should be active during vocalizations. To test this, we artificially evoked call-like vocalizations (EVOCs) under anesthesia using long trains of stimuli in DM (Vicario and Simpson 1995), and recorded from identified bursting locations in the contralateral PAm. For four of the five recorded PAm sites, we also simultaneously recorded neural activity in the contralateral HVC (ipsilateral to DM stimulation). EVOCs often outlasted the stimulus train by $100-200 \mathrm{~ms}$, allowing us to analyze data in the absence of any stimulus artifact. Because we were interested specifically in vocalization-related activity, and not in the short-latency trans-synaptic activation we described previously (Fig. 2B), we excluded data within the first $50 \mathrm{~ms}$ after the stimulus train offset. This prevented us from quantifying the temporal relationship between premotor activity in PAm and HVC, because premotor activity preceding the onset

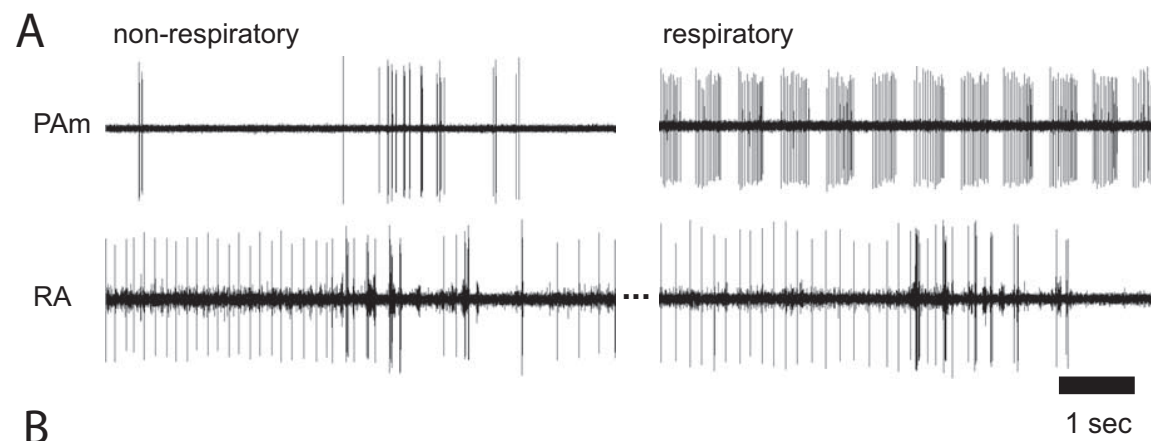

B

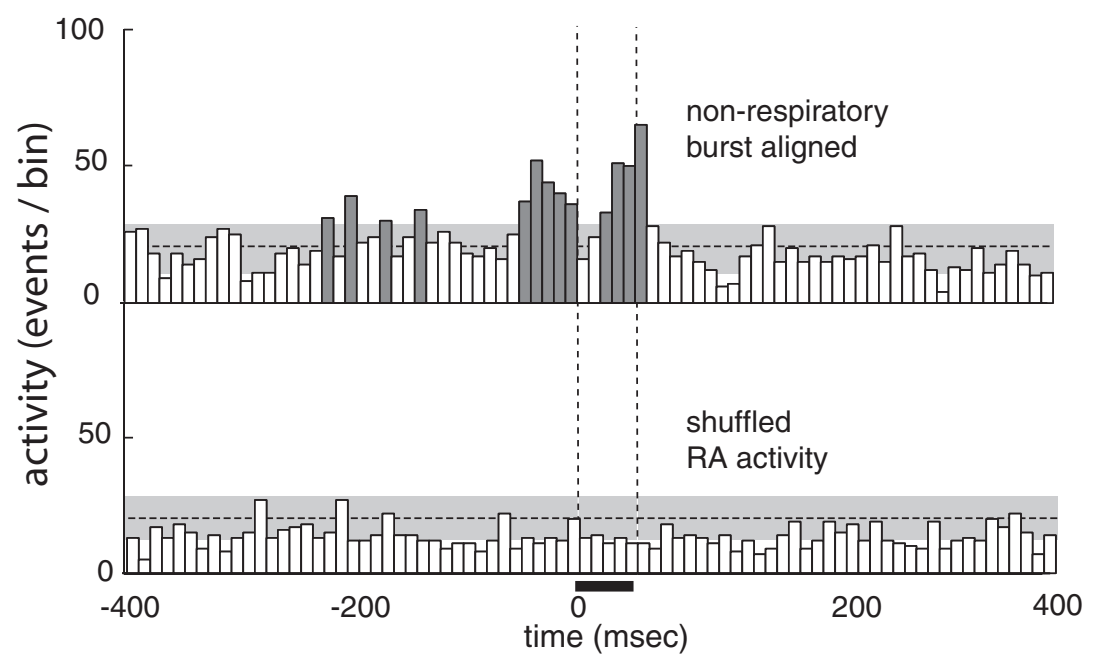

C
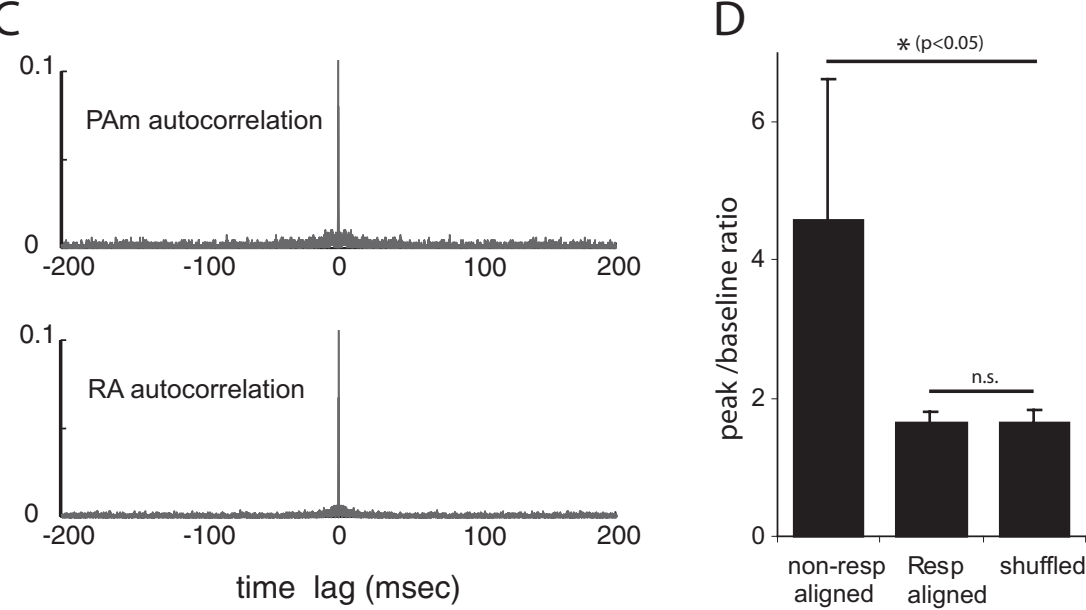

Figure 7. Spontaneous activity of nonrespiratory neurons in PAm is correlated with bursting in the contralateral RA. $\boldsymbol{A}_{\text {, }}$ Simultaneous recordings of two single units in the left PAm while maintaining the same unit in the contralateral RA. For the left pair, the recording electrode was placed closer to a site showing the characteristic nonrespiratory bursting pattern. Bursts in these neurons occur at approximately the same time as bursts (and subsequent pauses) in RA. In the right pair, a respiratory neuron recorded several minutes after the unit on the left shows a complete lack of correlated activity with the same RA neuron. $B$, Two PETHs are shown, representing activity recorded in the right RA of one bird. In the top PETH, RA activity was aligned to spikes from the nonrespiratory PAm neuron. In the bottom PETH, RA activity was randomly selected from the same recording. Significant peaks in the top histogram (dark gray) indicate that RA activity is high around the time of bursts in the PAm nonrespiratory bursting neurons, but shows no correlation with the shuffled control. The horizontal dotted lines indicate the mean height of histogram peaks generated from randomly selected $50 \mathrm{~ms}$ periods of RA activity. The gray areas indicate two SDs from that mean. The vertical dotted lines delineate the window of time for all PETHs used to calculate the values shown in $\boldsymbol{D}$. C, Autocorrelation plots of PAm (top) and RA (bottom) activity used in $\boldsymbol{B}$, showing values up to 0.1 (of 1.0). The absence of peaks at time lags greater or smaller than 0 indicate that the activity seen in the PETHs does not result from intrinsic correlation in RA or PAm. D, Comparison of peak/baseline ratios for RA activity $50 \mathrm{~ms}$ after alignment $(0-50 \mathrm{~ms})$ to nonrespiratory bursting neurons (left), and respiratory neurons (middle), and when the nonrespiratory aligned RA activity is randomly shuffled (right). Error bars indicate SD. Asterisks indicate significance by paired $t$ test. 
A

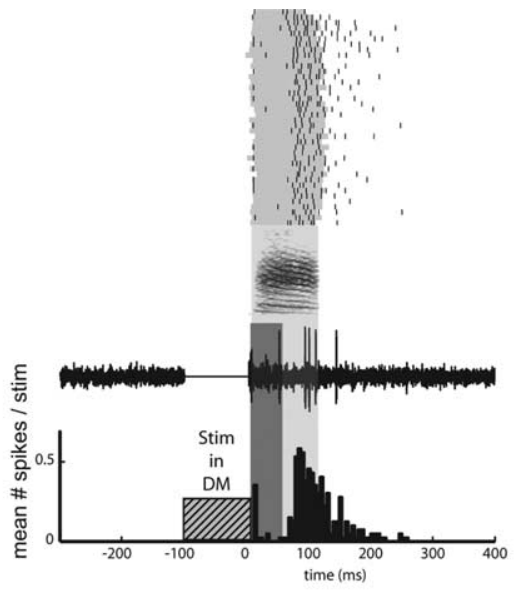

B

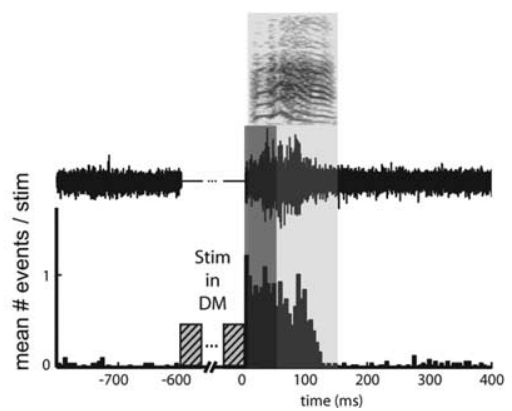

C

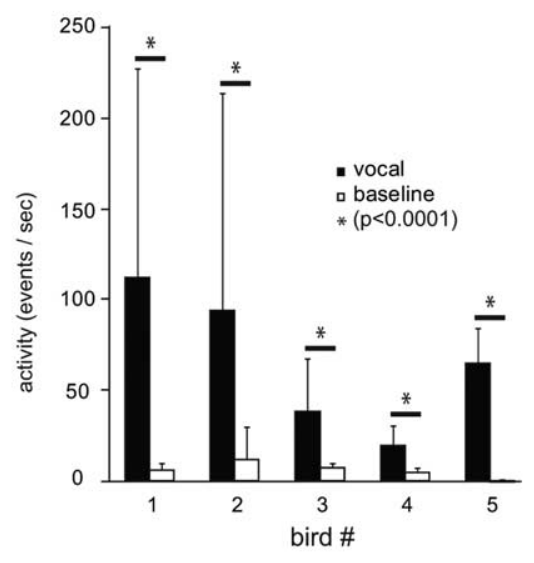

D

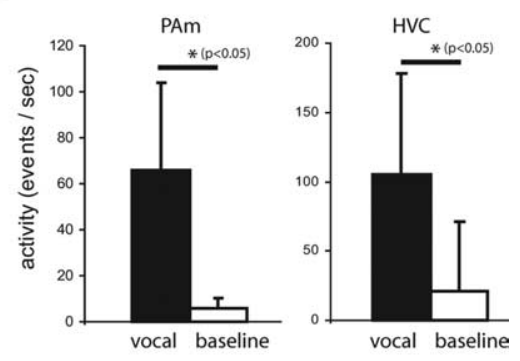

Figure 8. Nonrespiratory PAm neurons are active during vocalization evoked under anesthesia. $\boldsymbol{A}$, An example single-unit recording made from PAm. The raster at the top shows spike times (black) from one PAm neuron overlaid on periods of vocal production (gray) for 40 instances of DM stimulation-evoked vocalizations. The sonogram beneath the raster represents a sample vocalization. The neural trace below represents sample activity from the PAm neuron during that vocalization. The light gray box surrounding the sample indicates the vocalization period for this example. The PSTH below the trace was compiled from the 40 trials. To examine only vocalization-related activity and exclude activity resulting from direct trans-synaptic activation, the first 50 ms of activity after stimulation (dark gray box) was ignored when performing the analysis shown in $\mathbf{C}$ and $\boldsymbol{D}$. B, A multiunit example from another bird. A longer period of stimulation was used $(600 \mathrm{~ms})$ than in $\boldsymbol{A}$. Unlike in $\boldsymbol{A}$, activity at this site was elevated at an earlier time point relative to vocalization, indicating a heterogeneous temporal relationship between PAm nonrespiratory neurons and EVOC production. $C$, Mean \pm SD of neural activity in PAm recorded during multiple instances of DM stimulation evoked vocalizations (black) and paired baseline periods (immediately before each stimulation, white) for five birds. D, Population activity (grand mean \pm SD) recorded during evoked vocalizations for PAm (left) and HVC (right). PAm activity was gathered from five sites in four birds, and HVC activity was gathered from four sites in three birds. Asterisks indicate significance by paired $t$ test.

of these vocalizations was initiated during the stimulus period, but it allowed us to compare the overall level of activity in these two structures during the period of vocalization.

We found that activity of nonrespiratory bursting PAm neurons, as measured by overall activity rate (see Materials and Methods), was significantly higher during EVOCs than during periods of spontaneous activity recorded before stimulation for all five sites ( $p<0.0001$, paired $t$ tests; $n=49,20,34,38$, and 40 vocalizations, respectively) (Fig. $8 C$ ). Across the population, the mean activity rate was $65.7 \pm 38.3$ neural events/s during vocalizations, compared with $5.9 \pm 4.2$ events/s during baseline periods ( $p<0.05$, paired $t$ test; $n=5$ sites in 4 birds) (Fig. $8 D$ ). We found that HVC activity was also significantly elevated during EVOCs for all four recorded sites $(p<0.0001$, paired $t$ tests; $n=$ $49,20,34$, and 38). Across the population, the mean activity rate for HVC was $104.9 \pm 73.3$ during vocalization, and $21.4 \pm 23.5$ during baseline periods ( $p<0.05$, paired $t$ test; $n=4$ sites in 3 birds). Subjectively, the HVC activity resembled multiunit activity normally seen during spontaneous production of calls in the awake bird (a transient increase in activity associated with call production), suggesting that both DM stimulation-induced and natural vocalizations activate at least some of same areas of the vocal motor network. Interestingly, EVOC elicited neural responses in PAm neurons were not always sustained throughout the vocalization. Some sites appeared to be more active during the end of the vocalization (Fig. 8A) whereas others (Fig. $8 B$ ) were more responsive during the first half of the vocalization.

\section{Discussion}

Models of vocal motor production (Geschwindt, 1970; Yu and Margoliash, 1996; Margoliash, 1997), like most other models of motor control, place their emphasis on the forebrain (Tanji, 2001; Krauzlis, 2004). In contrast, the brainstem is viewed primarily as an output pathway for motor commands generated in the forebrain. During vocal production, however, respiratory feedback is likely to provide crucial information regarding peripheral respiratory demands during prolonged vocal activity. Consistent with this view, anatomical connections have been described between respiratory centers in the brainstem and forebrain vocal control centers. In mammals, the parabrachial area, a key vocal control structure (Jürgens, 2002; Smotherman et al., 2006), is known to receive ascending inputs from brainstem respiratory centers and send projections to cortical areas responsible for laryngeal control (Simonyan and Jürgens, 2005). In songbirds, robust bilateral projections connect the brainstem vocal respiratory centers PAm and DM with the thalamic relay nucleus Uva, which provides the primary input to nucleus HVC, a key forebrain vocal control nucleus (Nottebohm et al., 1982; Reinke and Wild, 1998; Striedter and $\mathrm{Vu}, 1998$; Coleman and $\mathrm{Vu}, 2005)$. HVC, in turn, projects ipsilaterally to these same brainstem areas via RA, completing a cyclic network. In this study we show in anesthetized adult male zebra finches that brief microstimulation in either PAm or DM can reliably evoke neural responses bilaterally in key vocal control areas of the forebrain. Furthermore, we show that stimulation of RA (or HVC) in one hemisphere can evoke robust responses in the contralateral RA. The ability of one or two current pulses to elicit reliable neural responses up to five synapses away with approximate latencies of 3-4 ms per synapse suggests the existence of a highly reliable and robust pathway by which the respiratory brainstem could influence forebrain vocal control systems.

The ability of Uva lesions to completely abolish responses in RA after brainstem stimulation demonstrates the crucial role for this thalamic nucleus in relaying ascending signals from the respiratory brainstem to forebrain vocal control centers. Previous evidence suggests that lesions of Uva lead to severe disruption of song in adult birds (Coleman and $\mathrm{Vu}, 2005$ ) by transforming the 
normally stereotyped sequences of syllables into long trains of repeated introductory notes that never transition to an ordered series of song syllables. These findings emphasize the critical nature of transmission of recurrent activity from brainstem vocal respiratory nuclei through Uva for song production. Because song is a sequenced behavior with high temporal precision (Cooper and Goller, 2004; Glaze and Troyer, 2006) produced by two separate forebrain hemispheres (Nottebohm et al., 1976; Williams et al., 1992; Wild, 1993; Vates et al., 1997; Reinke and Wild, 1998; Striedter and Vu, 1998; Wild et al., 2000), our current results are consistent with a model (Schmidt, 2003, 2004) whereby tightly coupled vocal-respiratory networks in both sides of the brainstem send precisely timed signals to both HVCs, via Uva, that are necessary for the initiation and continued production of song.

In the only well characterized example of a brainstem to forebrain contribution to motor control, projections from the superior colliculus have been suggested to convey corollary discharge signals regarding impending eye movements back to the frontal eye fields (Sommer and Wurtz, 2002, 2004a,b; Wurtz et al., 2005). Similar to the inability of birds to produce syllable sequences after Uva lesions, monkeys with lesions of the thalamic relay nucleus are impaired in their ability to perform saccade sequences even though single eye saccade performance is unaffected (Sommer and Wurtz, 2002). The similarity in these findings suggests that brainstem to forebrain projections in the song system might therefore play a similar role in relaying information regarding impending motor commands, or corollary discharge, from the respiratory brainstem to HVC.

It should be noted that the brainstem-forebrain pathway is not the only recurrent pathway present in the song system. RA also sends projections to the thalamic nucleus DMP, which projects bilaterally to MMAN, an ipsilateral forebrain input to HVC. Although disruption of this pathway only causes minor changes in vocal production (Foster and Bottjer, 2001), previous findings suggest that this pathway can compensate for the deleterious effects on song caused by Uva lesions (Coleman and $\mathrm{Vu}$, 2005). In the present study, we found that activity evoked by RA stimulation persisted in the contralateral RA even after lesions of Uva completely eliminated brainstem stimulation-evoked activity at the same RA recording sites. This result suggests that the MMAN pathway contributes to the evoked activity recorded in RA. This hypothesis was partially confirmed by the observed attenuation of RA to RA responses after MMAN lesions in birds that had already received Uva lesions. Combined Uva and MMAN lesions never completely eliminated the RA to RA response, probably because MMAN is a diffuse structure with poorly defined boundaries (Foster et al., 1997), and MMAN lesions were estimated to be incomplete in all cases. Nonetheless, the increased attenuation after combined MMAN and Uva lesions suggests that both nuclei mediate cross-hemisphere communication along their respective recurrent pathways.

In this study, we identified a novel subset of neurons in PAm whose intrinsic activity under anesthesia is roughly correlated with activity in the contralateral forebrain song system. Despite their proximity to inspiratory neurons, the PAm neurons we identified lack an intrinsic respiratory rhythm of their own, and instead exhibit nonrhythmic bursting activity which both follows and precedes moments of elevated bursting in the contralateral RA. Our evidence for a separate population of nonrespiratory neurons within PAm is supported by previous anatomical evidence of a subpopulation of neurons in PAm that send ascending projections to Uva (Wild, 2004). The ability of these neurons to burst is also consistent with the identification of neural cell types in the avian respiratory medulla with hyperpolarization-induced bursting properties (Kubke et al., 2005). The decrease in correlation between PAm and contralateral RA after Uva lesions suggests that nonrespiratory bursting PAm neurons may influence the timing of RA bursts through the ascending thalamic pathway. In addition, however, contralateral RA, by way of its many indirect projections back to PAm across the midline (Fig. 1), may in turn also influence burst times in PAm, a possibility suggested by the significant number of RA bursts that preceded those in PAm.

Using a paradigm to evoke vocalizations in anesthetized birds, we showed that nonrespiratory PAm neurons, which have low levels of spontaneous activity, become heavily recruited during vocalizations. The close spatial proximity of these neurons to neurons that are locked to the inspiratory phase of respiration, suggests that they might play an important role in integrating motor corollary discharge signals from RA during singing with information regarding the animal's respiratory state. Respiratory feedback in particular may provide crucial information about limitations at the periphery, such as the ability to maintain the high respiratory demands imposed by song (Wild et al., 1998), or the effect of song-related respiration on $\mathrm{O}_{2} / \mathrm{CO}_{2}$ exchange. Consistent with this view, $\mathrm{PAm}$ receives strong innervations from the nucleus tractus solitarius (Wild and Arends, 1987; Arends et al., 1988; Wild, 2004) which itself receives afferent inputs from the syrinx (Wild, 2004) and vagal nerve afferents that innervate intrapulmonary $\mathrm{CO}_{2}$ receptors in the lungs (Katz and Karten, 1983). Integration of this feedback with descending motor commands from RA may thus provide important signals for regulating both the tempo and duration of song.

Together, our current work suggests that the birdsong system could serve as an important model for deciphering how the brainstem integrates multiple input signals, and in turn influences forebrain motor output in a bottom-up manner. Because a crucial component of vocal production is the coordination between vocal output and respiration, the vocal respiratory brainstem might play a critical role in instructing the forebrain in all vocalization-related behaviors including human speech.

\section{References}

Arends JJA, Wild JM, Zeigler HP (1988) Projections of the nucleus of the tractus solitarius in the pigeon (Columba livia). J Comp Neurol 278:405-429.

Ashe J, Lungu OV, Basford AT, Lu XF (2006) Cortical control of motor sequences. Curr Opin Neurobiol 16:213-221.

Ashmore RC, Wild JM, Schmidt MF (2005) Brainstem and forebrain contributions to the generation of learned motor behaviors for song. J Neurosci 25:8543-8554.

Coleman MJ, Vu ET (2005) Recovery of impaired songs following unilateral but not bilateral lesions of nucleus uvaeformis of adult zebra finches. J Neurobiol 63:70-89.

Cooper BG, Goller F (2004) Multimodal signals: enhancement and constraint of song motor patterns by visual display. Science 303:544-546.

Douse MA, Duffin J (1992) Projections to Botzinger expiratory neurons by dorsal and ventral respiratory group neurons. NeuroReport 3:393-396.

Fee MS, Kozhevnikov AA, Hahnloser RHR (2004) Neural mechanisms of vocal sequence generation in the songbird. Ann NY Acad Sci 1016:153-170.

Feldman JL, Del Negro CA (2006) Looking for inspiration: new perspectives on respiratory rhythm. Nat Rev Neurosci 7:232-242.

Foster EF, Bottjer SW (2001) Lesions of a telencephalic nucleus in male zebra finches: influences on vocal behavior in juveniles and adults. J Neurobiol 46:142-165.

Foster EF, Mehta RP, Bottjer SW (1997) Axonal connections of the medial magnocellular nucleus of the anterior neostriatum in zebra finches. J Comp Neurol 382:364-381. 
Franz M, Goller F (2002) Respiratory units of motor production and song imitation in the zebra finch. J Neurobiol 51:129-141.

Geschwindt N (1970) The organization of language and the brain. Science 170:940-944.

Glaze CM, Troyer TW (2006) Temporal structure in zebra finch song: implications for motor coding. J Neurosci 26:991-1005.

Goldberg JM, Brown PB (1969) Response of binaural neurons of dog superior olivary complex to dichotic tonal stimuli: some physiological mechanisms of sound localization. J Neurophysiol 32:613-636.

Goller F, Cooper BG (2004) Peripheral motor dynamics of song production in the zehra finch. Ann NY Acad Sci 1016:130-152.

Hahnloser RHR, Kozhevnikov AA, Fee MS (2002) An ultra-sparse code underlies the generation of neural sequences in a songbird. Nature 419:65-70.

Jürgens U (2002) Neural pathways underlying vocal control. Neurosci Biobehav Rev 26:235-258.

Kalaska JF, Scott SH, Cisek P, Sergio LE (1997) Cortical control of reaching movements. Curr Opin Neurobiol 7:849-859.

Katz DM, Karten HJ (1983) Visceral representation within the nucleus of the tractus solitarius in the pigeon, Columba livia. J Comp Neurol 218:42-73.

Krauzlis RJ (2004) Recasting the smooth pursuit eye movement system. J Neurophysiol 91:591-603.

Kubke MF, Yazaki-Sugiyama Y, Mooney R, Wild JM (2005) Physiology of neuronal subtypes in the respiratory-vocal integration nucleus retroambigualis of the male zebra finch. J Neurophysiol 94:2379-2390.

Margoliash D (1997) Functional organization of forebrain pathways for song production and perception. J Neurobiol 33:671-693.

Nottebohm F, Stokes TM, Leonard CM (1976) Central control of song in the canary, Serinus canarius. J Comp Neurol 165:457-486.

Nottebohm F, Kelley DB, Paton JA (1982) Connections of vocal control nuclei in the canary telencephalon. J Comp Neurol 207:344-357.

Ramirez JM, Tryba AK, Pena F (2004) Pacemaker neurons and neuronal networks: an integrative view. Curr Opin Neurobiol 14:665-674.

Reinke H, Wild JM (1998) Identification and connections of inspiratory premotor neurons in songbirds and budgerigar. J Comp Neurol 391:147-163.

Schmidt MF (2003) Pattern of interhemispheric synchronization in HVC during singing correlates with key transitions in the song pattern. J Neurophysiol 90:3931-3949.

Schmidt MF, Ashmore RC, Vu ET (2004) Bilateral control and interhemispheric coordination in the avian song motor system. Ann NY Acad Sci 1016:171-186.

Simonyan K, Jürgens U (2005) Afferent subcortical connection into the motor cortical larynx area in the rhesus monkey. Neuroscience 130:119-131.

Smotherman M, Kobayasi K, Ma J, Zhang SY, Metzner W (2006) A mechanism for vocal-respiratory coupling in the mammalian parabrachial nucleus. J Neurosci 26:4860-4869.
Solis MM, Perkel DJ (2005) Rhythmic activity in a forebrain vocal control nucleus in vitro. J Neurosci 25:2811-2822.

Sommer MA, Wurtz RH (2002) A pathway in primate brain for internal monitoring of movements. Science 296:1480-1482.

Sommer MA, Wurtz RH (2004a) What the brain stem tells the frontal cortex. I. Oculomotor signals sent from superior colliculus to frontal eye field via mediodorsal thalamus. J Neurophysiol 91:1381-1402.

Sommer MA, Wurtz RH (2004b) What the brain stem tells the frontal cortex. II. Role of the SC-MD-FEF pathway in corollary discharge. J Neurophysiol 91:1403-1423.

Striedter GF, Vu ET (1998) Bilateral feedback projections to the forebrain in the premotor network for singing in zebra finches. J Neurobiol 34:27-40.

Suthers RA, Margoliash D (2002) Motor control of birdsong. Curr Opin Neurobiol 12:684-690.

Tanji J (2001) Sequential organization of multiple movements: involvement of cortical motor areas. Annu Rev Neurosci 24:631-651.

Vates GE, Vicario DS, Nottebohm F (1997) Reafferent thalamo-"cortical” loops in the song system of oscine songbirds. J Comp Neurol 380:275-290.

Vicario DS (1991) Organization of the zebra finch song control system: II. Functional organization of outputs from nucleus robustus archistriatalis. J Comp Neurol 309:486-494.

Vicario DS, Simpson HB (1995) Electrical stimulation in forebrain nuclei elicits learned vocal patterns in songbirds. J Neurophysiol 73:2602-2607.

Vu ET, Mazurek ME, Kuo YC (1994) Identification of a forebrain motor programming network for the learned song of zebra finches. J Neurosci 14:6924-6934.

Wild JM (1993) Descending projections of the songbird nucleus robustus archistriatalis. J Comp Neurol 338:225-241.

Wild JM (2004) Functional neuroanatomy of the sensorimotor control of singing. Ann NY Acad Sci 1016:438-462.

Wild JM, Arends JJ (1987) A respiratory-vocal pathway in the brain-stem of the pigeon. Brain Res 407:191-194.

Wild JM, Goller F, Suthers RA (1998) Inspiratory muscle activity during bird song. J Neurobiol 36:441-453.

Wild JM, Williams MN, Suthers RA (2000) Neural pathways for bilateral vocal control in songbirds. J Comp Neurol 423:413-426.

Williams H, Crane LA, Hale TK, Esposito MA, Nottebohm F (1992) Rightside dominance for song control in the zebra finch. J Neurobiol 23:1006-1020.

Wurtz RH, Sommer MA, Cavanaugh J (2005) Drivers from the deep: the contribution of collicular input to thalamocortical processing. In: Progress in brain research, Vol 149, Cortical function: a view from the thalamus (Casagrande VA, Guillery RW, Sherman SM, eds), pp 207-225. Amsterdam: Elsevier Science.

Yu AC, Margoliash D (1996) Temporal hierarchical control of singing in birds. Science 273:1871-1875. 